\section{Hydroxychloroquine is associated with lower platelet activity and improved vascular health in systemic lupus erythematosus}

\author{
MacIntosh Grant Cornwell (D) , ${ }^{1,2}$ Elliot S Luttrell-Williams, ${ }^{3}$ Michael Golpanian, ${ }^{4}$ \\ Hanane El Bannoudi, ${ }^{3}$ Khrystyna Myndzar, ${ }^{3}$ Peter Izmirly, ${ }^{4} \mathrm{H}$ Michael Belmont, ${ }^{4}$ \\ Stuart Katz, ${ }^{2}$ Nathaniel R Smilowitz (D) ${ }^{3}$ Alexis Engel, ${ }^{4}$ Robert Clancy, ${ }^{4}$ \\ Kelly Ruggles, ${ }^{1,2}$ Jill P Buyon, ${ }^{4}$ Jeffrey S Berger (1) ${ }^{3}$
}

To cite: Cornwell MG, Luttrell-Williams ES, Golpanian M, et al. Hydroxychloroquine is associated with lower platelet activity and improved vascular health in systemic lupus erythematosus. Lupus Science \& Medicine 2021;8:e000475. doi:10.1136/ lupus-2021-000475

MGC and ESL-W contributed equally.

JPB and JSB contributed equally.

Received 7 January 2021 Revised 10 February 2021 Accepted 20 February 2021
Check for updates

(C) Author(s) (or their employer(s)) 2021. Re-use permitted under CC BY-NC. No commercial re-use. See rights and permissions. Published by BMJ.

For numbered affiliations see end of article.

Correspondence to Dr Jeffrey S Berger; Jeffrey. Berger@nyumc.org

\section{ABSTRACT}

Objective Hydroxychloroquine (HCQ) is a mainstay of therapy in the treatment of SLE. The effect of HCQ on platelets and vascular health is uncertain. We investigated the relationship between HCQ use and dose with platelet activity, platelet transcriptomics and vascular health in patients with SLE.

Methods Platelet aggregation, platelet mRNA expression and vascular health (sublingual capillary perfused boundary region (PBR), red blood cell filling (RBCF) and brachial artery reactivity testing) were analysed by $\mathrm{HCQ}$ use and dose.

Results Among 132 subjects with SLE (age: $39.7 \pm 12.9$ years, $97 \%$ female), 108 were on HCQ. SLE disease activity was similar between subjects on and off $\mathrm{HCQ}$. Platelet aggregation in response to multiple agonists was significantly lower in patients on HCQ. There were inverse relationships between $\mathrm{HCQ}$ dose and gene expression pathways of platelet activity. Gene expression of $\mathrm{P}$ selectin (SELP) was inversely correlated with HCQ dose $(r=-0.41, p=0.003)$, which was validated at the protein level. Subjects on HCQ had improved vascular function correlating with $\mathrm{HCQ}$ dose as measured by lower PBR $(r=-0.52, p=0.007)$, higher RBCF $(r=0.55, p=0.004)$ and greater brachial artery reactivity $(r=0.43, p=0.056)$. Conclusion HCQ use was associated with decreased platelet activation and activation-related transcripts and improved vascular health in SLE.

\section{INTRODUCTION}

SLE is an autoimmune disease characterised by a wide array of symptoms that can affect virtually all organ systems. The antimalarial medication hydroxychloroquine (HCQ) is the most frequently prescribed long-term medication used to treat this disease, a practice driven by extensive literature supporting its benefits in patients with SLE. 'Notably, HCQ has been associated with several benefits in patients with SLE, including a decrease in the rate of disease flares, ${ }^{2}$ protection against

\section{Key messages}

What is already known about this subject?

- Hydroxychloroquine (HCQ) is a mainstay of therapy in patients with SLE.

What does this study add?

- Subjects with SLE taking HCQ exhibited reduced platelet aggregation and improved vascular health.

- Platelet RNA expression revealed that platelet functional pathways were downregulated in patients on $\mathrm{HCQ}$, and that the expression of these pathways decreased with increasing $\mathrm{HCQ}$ dose.

How might this impact on clinical practice or future developments?

- $\mathrm{HCQ}$ is associated with lower platelet activity and improved vascular health, providing important pleiotropic mechanisms of benefit for HCQ in SLE.

organ damage, ${ }^{3}$ improvement in overall survival $^{4}$ and reduction in the risk of thrombosis. ${ }^{25}$ Although antithrombotic effects have been demonstrated in clinical studies, the underlying mechanisms driving this protective effect are not fully understood. Accordingly, we compared platelet phenotypes and measures of vascular health in patients with SLE who were and were not prescribed HCQ to define the cardiovascular effects of this therapy in SLE.

\section{METHODS}

Subjects with SLE (meeting at least four of the revised American College of Rheumatology (ACR) or Systemic Lupus International Collaborating Clinics criteria) ${ }^{67}$ were recruited into an ongoing NIH-funded study investigating platelet activity, transcriptome and vascular health. Individuals who used aspirin or non-steroidal anti-inflammatory 
drugs or had a platelet count $<100 \times 10^{9} / \mathrm{L}$ at the time of blood collection were excluded. Information including medication use, weight and other patient history was recorded at the time of enrolment. Blood was collected for haematology analysis using the Sysmex XN-1000 analyser, platelet aggregation via the Helena AggRAM system, and platelet RNA isolation and storage via magnetic separation using the StemCell kit to deplete CD45+ and GlyA+ cells. Microvascular function was assessed via sublingual sidestream darkfield imaging, and brachial artery reactivity testing was used to evaluate large vessel function. Stored RNA was isolated and analysed by RNA sequencing (Illumina HiSeq 4000 sequencing). FASTQ files from RNA sequencing were processed using the Seq-N-Slide pipeline. Reads were aligned to the hg38 genome using STAR V.2.6. $1^{8}$ and quantified using featureCounts V.1.6.3. ${ }^{9}$

All downstream analyses were performed in R V.3.6.1. Differential expression analysis was performed via DESeq2 V.1.24. Weighted gene coexpression network analysis (WGCNA) was performed using the WGCNA package enriching for gene sets provided via msigdbr V.7.0.1. Multiple hypothesis correction was done using the Benjamini-Hochberg method. Heatmaps were created using ComplexHeatmap V.2.0.0 and all plotting were done using ggplot2 V.3.2.1. Flow cytometry was performed using a BD Accuri C6 flow cytometer and a Miltenyi Biotec MACSQuant Analyzer. All pairwise comparisons were analysed using non-parametric Wilcoxon tests. Correlational analyses were performed using non-parametric Spearman tests. For all correlational analyses exploring dose response with regard to phenotypic metric, both the correlational values for the entire data set and the Wilcoxon $\mathrm{p}$ value for no HCQ versus HCQ were included.

\section{RESULTS}

Among 132 subjects with SLE, 108 were on HCQ. The reasons patients were not on HCQ included ocular concerns, gastrointestinal discomfort, rash and patient refusal. The mean age of the entire cohort was $39.7 \pm 12.9$ and $97 \%$ were female. Lupus disease activity at the time of blood draw assessed by the HYBRID SELENA Systemic Lupus Erythematosus Disease Activity Index (SLEDAI), a modification of the original SELENA SLEDAI, ${ }^{10}$ in which proteinuria UPCR (Urine ProteinCreatinine Ratio) $>0.5 \mathrm{~g} / 24$ hours is always counted on the renal domain, was 3.58 (range 0-20). Demographics and overall SLE ACR classification criteria and SLEDAI at the time of blood draw did not differ between those on and off HCQ, except for ulcers for the former and alopecia and pyuria for the latter (table 1). Platelet count and size were not different between groups (figure 1A). Since platelet aggregation in response to submaximal agonist concentration is a useful proxy for platelet hyperreactivity, we assessed platelet aggregation in subjects on and off HCQ. In response to submaximal ADP at multiple concentrations, platelet aggregation was lower in patients on HCQ versus those not prescribed HCQ (figure 1B).
There was an inverse relationship between HCQ dosing and platelet aggregation in response to ADP (shown: $1 \mu \mathrm{M}$ : $\mathrm{r}=-0.27, \mathrm{p}=0.008$; figure $1 \mathrm{C}$; online supplemental figure 1A). Since aspirin use was an exclusion criterion, aggregation in response to arachidonic acid (AA) was robust and similar between groups. However, after incubating platelets with aspirin $(3 \mathrm{mM})$ in vitro, platelet aggregation in response to AA was lower in the HCQ group compared with the non-HCQ group ( $\mathrm{p}=0.017$; figure $1 \mathrm{~B})$.

To investigate potential mechanisms of HCQ relating to decreased platelet aggregation, we evaluated platelet RNA sequencing on samples from 49 patients (41 on HCQ, 8 not on HCQ). Using WGCNA's eigengene functionality and Gene Ontology (GO) terms from MsigDB, we reduced platelet-specific pathway expression on a per sample basis to a single eigengene. These values were then correlated for each pathway with HCQ use, dose and dose-adjusted for weight. Several pathways were negatively correlated with HCQ dosing, including most significantly platelet dense granule membrane $(r=-0.42, p<0.01)$ and positive regulation of platelet activation $(\mathrm{r}=-0.37, \mathrm{p}=0.01$; figure 1D). Positive regulation of platelet activation was inversely related to HCQ, especially when dosing reached levels above the recommended $5 \mathrm{mg} / \mathrm{kg}$ (figure $1 \mathrm{E}$ ).

The gene encoding for P-selectin (SELP) was a major contributor to both of these pathways. The expression of SELP negatively correlated $(\mathrm{r}=-0.41, \mathrm{p}=0.003$; figure $1 \mathrm{~F})$ with HCQ dose/weight. To validate this finding, P-selectin surface expression in response to thrombin agonist was measured via flow cytometry and reported as a ratio of basal P-selectin expression. Subjects on HCQ had a significantly reduced fold change in P-selectin expression compared with subjects not taking HCQ ( $\mathrm{p}=0.037$; figure $1 \mathrm{G}$; online supplemental figure $1 \mathrm{~B}$ ) and this correlated with HCQ dose/weight, although not quite reaching a significance level of $\mathrm{p}<0.05(\mathrm{r}=-0.29, \mathrm{p}=0.066$; figure $1 G)$.

Finally, we investigated vascular function in subjects on and off HCQ. Subjects on HCQ had improved microvascular function compared with patients who were not taking HCQ as demonstrated by an increased proportion of sublingual capillaries filled with red blood cells $(\mathrm{p}=0.014)$ and smaller perfused boundary region (PBR, $\mathrm{p}=0.042)$. HCQ dosing correlated with both parameters of vascular function $(\mathrm{r}=-0.52, \mathrm{p}=0.007$ for $\mathrm{PBR}$, figure $1 \mathrm{H}$; Online supplemental figure $1 \mathrm{C} ; \mathrm{r}=0.55, \mathrm{p}=0.004$, red blood cell fillings, figure 1I; online supplemental figure 1D). Brachial artery reactivity testing also trended positively with $\mathrm{HCQ}$ dose/weight $(\mathrm{r}=0.43, \mathrm{p}=0.056$; figure $1 \mathrm{~J}$; online supplemental figure $1 \mathrm{E}$ ).

\section{DISCUSSION}

Overall, patients with SLE taking HCQ displayed reduced platelet aggregation and improved metrics of microvascular and large vessel function when compared with those with similar SLE manifestations not on HCQ. Furthermore, these effects appear to be dose-dependent, 
Table 1 Demographic and lupus characterisation of cohorts

\begin{tabular}{|c|c|c|c|c|c|c|c|}
\hline & $\begin{array}{l}\text { All } \\
(\mathrm{N}=132)\end{array}$ & $\begin{array}{l}H C Q \\
(n=108)\end{array}$ & $\begin{array}{l}\text { No HCQ } \\
(n=24)\end{array}$ & $P$ value & $\begin{array}{l}\text { HCQ RNA-seq } \\
(n=41)\end{array}$ & $\begin{array}{l}\text { No HCQ RNA-seq } \\
(n=8)\end{array}$ & $P$ value \\
\hline Age, mean (SD) & 39.7 (12.9) & $39.4(12.7)$ & $40.8(14.2)$ & 0.66 & $40.9(12.2)$ & $44.9(14.0)$ & 0.47 \\
\hline Female, n (\%) & $128(97.0)$ & 104 (96.3) & $24(100)$ & 0.04 & $41(100)$ & $8(100)$ & 1 \\
\hline \multicolumn{8}{|l|}{ Race, n (\%) } \\
\hline Asian & $27(20.5)$ & $25(23.1)$ & $2(8.3)$ & 0.04 & $10(24.4)$ & $1(12.5)$ & 0.42 \\
\hline Black or African American & $41(31.1)$ & $33(30.6)$ & $8(33.3)$ & 0.8 & $10(24.4)$ & $0(0)$ & $>0.01$ \\
\hline White & $59(44.7)$ & $46(42.6)$ & $13(54.2)$ & 0.32 & $20(48.8)$ & $6(75.0)$ & 0.18 \\
\hline Other/unknown & $5(3.7)$ & $4(3.7)$ & $1(4.2)$ & 1 & $1(2.4)$ & $1(12.5)$ & 0.33 \\
\hline \multicolumn{8}{|l|}{ Ethnicity, n (\%) } \\
\hline Hispanic or Latino & $49(37.1)$ & 37 (34.3) & $12(50.0)$ & 0.18 & $18(43.9)$ & $5(62.5)$ & 0.37 \\
\hline \multicolumn{8}{|c|}{ SELENA SLEDAI domains* (\%) } \\
\hline Seizure & $0(0)$ & $0(0)$ & $0(0)$ & 1 & $0(0)$ & $0(0)$ & 1 \\
\hline Psychosis & $0(0)$ & $0(0)$ & $0(0)$ & 1 & $0(0)$ & $0(0)$ & 1 \\
\hline Organic brain syndrome & $0(0)$ & $0(0)$ & $0(0)$ & 1 & $0(0)$ & $0(0)$ & 1 \\
\hline Visual disturbance & $0(0)$ & $0(0)$ & $0(0)$ & 1 & $0(0)$ & $0(0)$ & 1 \\
\hline Cranial nerve disorder & $0(0)$ & $0(0)$ & $0(0)$ & 1 & $0(0)$ & $0(0)$ & 1 \\
\hline Lupus headache & $0(0)$ & $0(0)$ & $0(0)$ & 1 & $0(0)$ & $0(0)$ & 1 \\
\hline Cerebrovascular accident & $0(0)$ & $0(0)$ & $0(0)$ & 1 & $0(0)$ & $0(0)$ & 1 \\
\hline Vasculitis & $0(0)$ & $0(0)$ & $0(0)$ & 1 & $0(0)$ & $0(0)$ & 1 \\
\hline Arthritis & $2(1.5)$ & $1(1.0)$ & $1(4.2)$ & 0.45 & $1(2.4)$ & $1(12.5)$ & 0.45 \\
\hline Myositis & $0(0)$ & $0(0)$ & $0(0)$ & 1 & $0(0)$ & $0(0)$ & 1 \\
\hline Haematuria & $4(3.3)$ & $2(2.1)$ & $2(8.7)$ & 0.29 & $2(5.4)$ & $2(25)$ & 0.28 \\
\hline Proteinuria & $29(22.0)$ & $24(23.8)$ & $5(21.7)$ & 0.84 & $12(30.8)$ & $2(25)$ & 0.76 \\
\hline Pyuria & $5(3.8)$ & $5(5.1)$ & $0(0)$ & 0.02 & $3(7.9)$ & $0(0)$ & 0.08 \\
\hline Rash & $20(15.2)$ & 15 (13.9) & $5(20.8)$ & 0.45 & $7(17.1)$ & $1(12.5)$ & 0.75 \\
\hline Alopecia & $10(7.6)$ & $7(6.5)$ & $3(12.5)$ & 0.42 & $4(9.8)$ & $0(0)$ & 0.04 \\
\hline Mucosal ulcers & $2(1.5)$ & $1(1)$ & $1(4.2)$ & 0.45 & $0(0)$ & $0(0)$ & 1 \\
\hline Pleurisy & $2(1.5)$ & $0(0$ & $2(8.3)$ & 0.16 & $0(0)$ & $0(0)$ & 1 \\
\hline Pericarditis & $0(0)$ & $0(0)$ & $0(0)$ & 1 & $0(0)$ & $0(0)$ & 1 \\
\hline Low complement & $54(40.9)$ & $43(41.7)$ & $11(45.8)$ & 0.72 & $21(51.2)$ & $3(37.5)$ & 0.51 \\
\hline Anti-dsDNA & $49(37.1)$ & 39 (39.4) & $10(45.5)$ & 0.62 & $17(43.6)$ & $4(57.1)$ & 0.55 \\
\hline Fever & $0(0)$ & $0(0)$ & $0(0)$ & 1 & $0(0)$ & $0(0)$ & 1 \\
\hline Thrombocytopaenia & $4(3.0)$ & $2(2.0)$ & $2(8.7)$ & 0.28 & $2(5.0)$ & $1(12.5)$ & 0.58 \\
\hline Mean total SLEDAI (SD) & $3.58(3.56)$ & $3.45(3.47)$ & 4.09 (3.99) & 0.5 & $4.41(4.32)$ & $5.29(4.79)$ & 0.67 \\
\hline \multicolumn{8}{|l|}{ ACR criteria (\%) } \\
\hline Malar rash & $67(50.8)$ & $56(51.9)$ & $11(45.8)$ & 0.6 & $21(51.2)$ & $5(62.5)$ & 0.58 \\
\hline Discoid rash & $30(22.7)$ & 23 (21.3) & 7 (29.2) & 0.45 & $12(29.3)$ & $3(37.5)$ & 0.68 \\
\hline Photosensitivity & $49(37.1)$ & 39 (36.1) & $10(41.7)$ & 0.63 & $10(24.4)$ & $5(62.5)$ & 0.08 \\
\hline Oral ulcers & 25 (19.1) & $18(16.8)$ & $7(29.2)$ & 0.23 & $3(7.3)$ & $5(62.5)$ & 0.02 \\
\hline Arthritis & $99(75)$ & $80(74.1)$ & 19 (79.2) & 0.59 & 30 (73.2) & $6(75)$ & 0.92 \\
\hline Pleuritis & $22(16.7)$ & $16(14.8)$ & $6(25)$ & 0.3 & $9(22)$ & $2(25)$ & 0.87 \\
\hline Pericarditis & 24 (18.2) & 19 (17.6) & $5(20.8)$ & 0.73 & $8(19.5)$ & $2(25)$ & 0.76 \\
\hline Proteinuria & $63(47.7)$ & $52(48.1)$ & $10(41.7)$ & 0.84 & $22(53.7)$ & $3(37.5)$ & 0.44 \\
\hline Seizures & $4(3)$ & $3(2.8)$ & $1(4.2)$ & 0.76 & $1(2.4)$ & $0(0)$ & 0.32 \\
\hline Psychosis & $3(2.3)$ & $2(1.9)$ & $1(4.2)$ & 0.6 & $0(0)$ & $0(0)$ & 1 \\
\hline
\end{tabular}


Table 1 Continued

\begin{tabular}{|c|c|c|c|c|c|c|c|}
\hline & $\begin{array}{l}\text { All } \\
(\mathrm{N}=132)\end{array}$ & $\begin{array}{l}\text { HCQ } \\
(n=108)\end{array}$ & $\begin{array}{l}\text { No HCQ } \\
(n=24)\end{array}$ & $P$ value & $\begin{array}{l}\text { HCQ RNA-seq } \\
(n=41)\end{array}$ & $\begin{array}{l}\text { No HCQ RNA-seq } \\
(n=8)\end{array}$ & $P$ value \\
\hline Haemolytic anaemia & $8(6.1)$ & $7(6.5)$ & $1(4.2)$ & 0.63 & $1(2.4)$ & $0(0)$ & 0.32 \\
\hline Leucopenia & 58 (43.9) & $49(45.4)$ & $9(37.5)$ & 0.49 & $17(41.5)$ & $2(25)$ & 0.38 \\
\hline Lymphopaenia & $60(45.5)$ & $51(47.2)$ & $9(37.5)$ & 0.39 & 24 (58.5) & $2(25)$ & 0.09 \\
\hline Thrombocytopaenia & $18(13.6)$ & $14(13)$ & $4(16.7)$ & 0.66 & $6(14.6)$ & $3(37.5)$ & 0.26 \\
\hline Anti-dsDNA & $99(75)$ & $80(74.1)$ & $19(79.2)$ & 0.59 & $30(73.2)$ & $6(75)$ & 0.92 \\
\hline Anti-Smith & $53(40.2)$ & $45(41.7)$ & $8(33.3)$ & 0.45 & $20(48.8)$ & $3(37.5)$ & 0.58 \\
\hline Antiphospholipid & $29(22)$ & $14(13)$ & $6(25)$ & 0.22 & $7(17.1)$ & $1(12.5)$ & 0.75 \\
\hline ANA & 123 (93.2) & $108(100)$ & $24(100)$ & 1 & $41(100)$ & $8(100)$ & 1 \\
\hline
\end{tabular}

${ }^{*}$ Percentages and statistical testing were performed for each SLEDAI variable using the available denominator. SLEDAI values were available for $>90 \%$ of patients.

ACR, American College of Rheumatology; anti-dsDNA, anti-double-stranded DNA; HCQ, hydroxychloroquine; SLEDAI, Systemic Lupus Erythematosus Disease Activity Index.

especially when HCQ was prescribed above the recommended dose of $5 \mathrm{mg} / \mathrm{kg}$. The analysis of platelet transcriptomic data confirmed these results by revealing that platelet activation pathways were negatively correlated with HCQ use, dose and HCQ-adjusted dose/weight.

Given the importance of proper dose per weight to the prescribing of HCQ we focused results on the correlations between transcriptional expression and the dose/ weight ratio. Two pathways that were significantly negatively correlated with HCQ dose/weight were platelet dense granule membrane and positive regulation of platelet activation. These pathways are both related specifically to the composition of the active components in platelets, and the activation of platelets for cell-cell communication and aggregation. P-selectin (SELP) is a major contributor to both of these pathways' transcriptional profiles. Indeed, P-selectin transcription revealed a negative correlation with dose/weight $(\mathrm{r}=-0.41$, $\mathrm{p}=0.0031$ ). Consistently, a reduced fold change in P-selectin surface expression was observed in activated platelets from patients on HCQ. P-selectin has been shown to play significant roles in platelet adhesion to endothelial cells and immune cells, and is directly related to platelet activation, coagulation and vascular health. ${ }^{11}$ The connections between P-selectin and SLE have not been extensively explored. Studies have shown that variations in the SELP gene have been identified to confer higher risk of developing SLE in patients, although through an unknown mechanism. ${ }^{12}$ Furthermore, recent studies in mouse models of SLE have demonstrated that P-selectin is upregulated in mice with lupus nephritis and treating mice with P-selectin blocking antibodies improved outcome and reduced renal vascular damage and hypoxic injury. ${ }^{13}$

HCQ as an antithrombotic has been described previously and patients with SLE are noted to have fewer thrombotic events when treated with HCQ. ${ }^{25} \mathrm{HCQ}$ has been shown to reduce platelet aggregation in the setting of collagen and ADP-induced aggregation. ${ }^{14}$ In addition,
HCQ has also been shown to affect the rheology of blood by inhibiting red blood cell aggregation. ${ }^{14}$ To our knowledge, the relationship between platelet transcriptomics and HCQ exposure has never been described, and our findings support previously established clinical effects of HCQ.

Several limitations of this study are acknowledged. A majority of patients were prescribed HCQ consistent with given current guidelines for the treatment of SLE, but this leads to a significant difference in the number of patients in each cohort. Although gene expression differences between subjects on and off $\mathrm{HCQ}$ were less significant than the results seen from dose-response correlations, the sample size of those subjects not on HCQ was limited, therefore reducing our statistical power. Reasons for not taking HCQ included ocular concerns, gastrointestinal discomfort, rash and patient declination. The use of medications that could influence platelet reactivity precluded participation by patients, particularly those with active arthritis. HCQ blood levels were not available. The absence of HCQ levels may overinterpret the presumption of HCQ effects since it is well described that adherence to the drug is a management issue despite continued reinforcement of compliance. While not mitigating having blood levels, we at least attempted to address levels by considering HCQ dose normalised by weight. Moreover, given concerns for retinal toxicity, dosing may often be reduced in stable patients and thus analysis by $\mathrm{mg} / \mathrm{kg}$ prescribed was also done to see whether HCQ conferred favourable vascular effects even at low prescribed doses. ${ }^{15}$ Finally, the associations between HCQ and platelet activity were observed in a cross-sectional analysis and an effect on long-term cardiovascular outcomes cannot necessarily be implied.

In aggregate, data from this study demonstrate a dose-dependent association between HCQ and platelet activity, transcriptome and overall vascular health. These results reinforce the antithrombotic properties of HCQ in patients with SLE and potential protective benefit in 

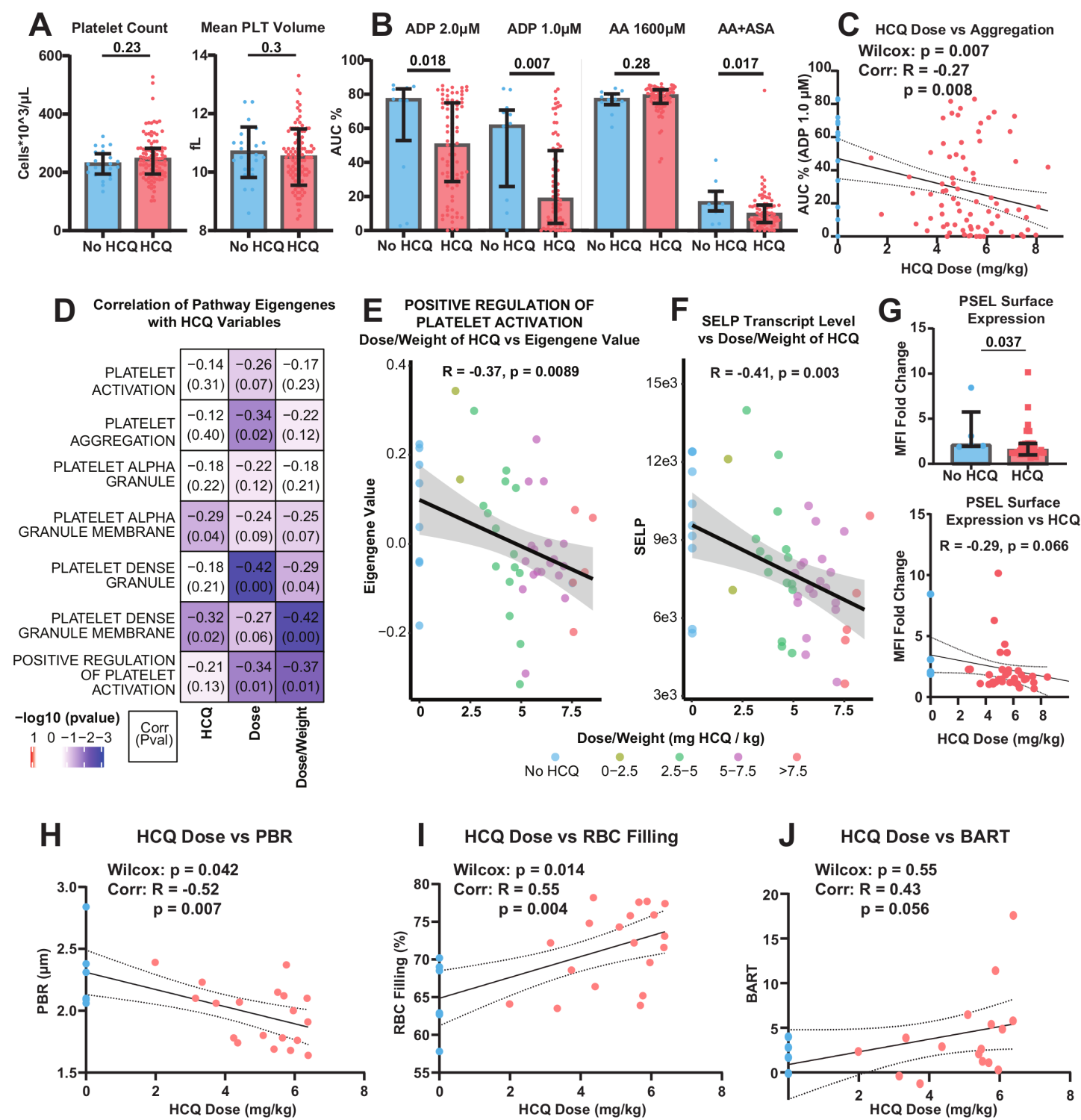

Figure 1 Associations between $\mathrm{HCQ}$ use and dose and platelet function, transcriptomics and vascular function. Comparison of $\mathrm{HCQ}$ and non-HCQ groups of subjects with SLE examining (A) platelet count and size (Mean PLT Volume). (B) Aggregation in response to ADP and AA with and without in vitro aspirin (ASA). (C) Spearman correlation of daily HCQ dosing and aggregation in response to $1 \mu \mathrm{M} A D P$ as measured by the area under the curve (AUC). The respective pairwise comparison of no $H C Q$ versus $\mathrm{HCQ}$ is also noted with a Wilcoxon $p$ value. (D) Heatmap depicting the correlation and $p$ value between the sample eigengene values associated with platelet pathways and HCQ use, dose and dose-adjusted for weight. (E) Scatterplot showing the eigengene values for the positive regulation of platelet activation gene set compared against HCQ dose/weight for each patient. (F) Scatterplot showing the normalised transcript level of SELP compared against HCQ dose/weight for each patient. (G) Comparison of P-selectin (PSEL) surface expression fold change in subjects on and off HCQ as measured by flow cytometry and a Spearman correlation of HCQ dose and P-selectin fold change. Mean Fluorescence Intensity (MFI) values are reported. Spearman correlations of daily HCQ dosing and PBR $(H), R B C$ filling $(I)$ and BART $(J)$ were also examined, shown here with $95 \% \mathrm{Cl}$ bands. The respective pairwise comparisons of no $\mathrm{HCQ}$ versus $\mathrm{HCQ}$ are also noted with a Wilcoxon $p$ value. AA, arachidonic acid; BART, brachial artery reactivity testing; $\mathrm{HCQ}$, hydroxychloroquine; PBR, perfused boundary region; RBC, red blood cell; SELP, P-selectin.

a disease where premature atherosclerosis is a significant comorbidity.

\section{Author affiliations}

${ }^{1}$ Institute for Systems Genetics, NYU Langone Health, New York City, New York, USA ${ }^{2}$ Division of Translational Medicine, New York University School of Medicine, New York City, New York, USA

${ }^{3}$ Division of Cardiology, Department of Medicine, New York University Grossman School of Medicine, New York City, New York, USA
${ }^{4}$ Division of Rheumatology, Department of Medicine, New York University School of Medicine, New York City, New York, USA

Acknowledgements The authors thank the individuals who agreed to participate in this trial and the staff of the NYU Langone facilities that enabled this work to be done.

Contributors MGC, ESL-W and MG wrote the initial draft of the paper. ESL-W and $\mathrm{HEB}$ performed the experiments related to platelet behaviour and isolated the RNA from the platelets. MG and AE collected and curated the clinical data for 
the patients. MGC performed the bioinformatic processing and analysis for the RNA sequencing. KM, PI, HMB, SK, NS, RC and KR all provided key insights for the overall project and contributed to and edited the manuscript. JPB and JB oversaw the experiments, clinical collection and analytical processes. All authors edited, contributed to and approved the final manuscript.

Funding This work was supported by the National Institutes of Health (grant R01HL139909, JPB and JB; grant T32GM136573, MGC) and National Institute of Arthritis and Musculoskeletal and Skin Diseases, NIH (grant P50-AR-07059, JPB and PI).

\section{Competing interests None declared.}

Patient and public involvement Patients and/or the public were involved in the design, or conduct, or reporting, or dissemination plans of this research. Refer to the Methods section for further details.

Patient consent for publication Not required.

Ethics approval The study was reviewed and approved by the NYU Langone Data and Safety Monitoring Board.

Provenance and peer review Not commissioned; externally peer reviewed. Data availability statement Data are available upon reasonable request.

Open access This is an open access article distributed in accordance with the Creative Commons Attribution Non Commercial (CC BY-NC 4.0) license, which permits others to distribute, remix, adapt, build upon this work non-commercially, and license their derivative works on different terms, provided the original work is properly cited, appropriate credit is given, any changes made indicated, and the use is non-commercial. See: http://creativecommons.org/licenses/by-nc/4.0/.

\section{ORCID iDs}

MacIntosh Grant Cornwell http://orcid.org/0000-0002-3041-4410

Nathaniel R Smilowitz http://orcid.org/0000-0002-6823-2815

Jeffrey S Berger http://orcid.org/0000-0001-8216-4647

\section{REFERENCES}

1 Ruiz-Irastorza G, Ramos-Casals M, Brito-Zeron P, et al. Clinical efficacy and side effects of antimalarials in systemic lupus erythematosus: a systematic review. Ann Rheum Dis 2010;69:20-8.
2 Ruiz-Irastorza G, Egurbide MV, Pijoan Jl, et al. Effect of antimalarials on thrombosis and survival in patients with systemic lupus erythematosus. Lupus 2006;15:577-83.

3 Fessler BJ, Alarcón GS, McGwin G, et al. Systemic lupus erythematosus in three ethnic groups: XVI. Association of hydroxychloroquine use with reduced risk of damage accrual. Arthritis Rheum 2005;52:1473-80.

4 Alarcón GS, McGwin G, Bertoli AM, et al. Effect of hydroxychloroquine on the survival of patients with systemic lupus erythematosus: data from LUMINA, a multiethnic US cohort (LUMINA L). Ann Rheum Dis 2007;66:1168-72.

5 Ho KT, Ahn CW, Alarcón GS, et al. Systemic lupus erythematosus in a multiethnic cohort (LUMINA): XXVIII. factors predictive of thrombotic events. Rheumatology 2005;44:1303-7.

6 Hochberg MC. Updating the American College of rheumatology revised criteria for the classification of systemic lupus erythematosus. Arthritis Rheum 1997;40:1725.

7 Petri M, Orbai A-M, Alarcón GS, et al. Derivation and validation of the systemic lupus international collaborating clinics classification criteria for systemic lupus erythematosus. Arthritis Rheum 2012;64:2677-86.

8 Dobin A, Davis CA, Schlesinger F, et al. Star: ultrafast universal RNAseq aligner. Bioinformatics 2013;29:15-21.

9 Liao Y, Smyth GK, Shi W. featureCounts: an efficient General purpose program for assigning sequence reads to genomic features. Bioinformatics 2014;30:923-30.

10 Petri M, Kim MY, Kalunian KC, et al. Combined oral contraceptives in women with systemic lupus erythematosus. $N$ Engl J Med Overseas Ed 2005;353:2550-8.

11 George R, Bhatt A, Narayani J, et al. Enhanced P-selectin expression on platelet-a marker of platelet activation, in young patients with angiographically proven coronary artery disease. Mol Cell Biochem 2016;419:125-33.

12 Morris DL, Graham RR, Erwig L-P, et al. Variation in the upstream region of P-selectin (SELP) is a risk factor for SLE. Genes Immun 2009;10:404-13.

13 Zhang L, Chen S, Liu Y, et al. P-Selectin blockade ameliorates lupus nephritis in MRL/lpr mice through improving renal hypoxia and evaluation using BOLD-MRI. J Trans/ Med 2020;18:116.

14 Belizna C. Hydroxychloroquine as an anti-thrombotic in antiphospholipid syndrome. Autoimmun Rev 2015;14:358-62.

15 Costedoat-Chalumeau N, Houssiau F, Izmirly P, et al. A prospective international study on adherence to treatment in 305 patients with flaring SLE: assessment by drug levels and self-administered questionnaires. Clin Pharmacol Ther 2019;106:374-82. 